\title{
Possible geomagnetic activity effects on weather
}

\author{
J. Bochníček, P. Hejda, V. Bucha, J. Pýcha \\ Geophysical Institute, Academy of Science Czech Republic, Boční II, 14131 Prague, Czech Republic \\ E-mail: jboch@ig.cas.cz
}

Received: 16 June 1998 / Revised: 18 November 1998 / Accepted: 20 November 1998

\begin{abstract}
Tropospheric temperature and pressure fields on the Northern Hemisphere in the winter periods 19521996 were investigated. Composite maps of those fields, created for the high and low geomagnetic activity and individual quasi-biennial oscillation (QBO), phases show clear differences not only between different levels of geomagnetic activity, but also between the two phases of QBO. Special attention was given to the behaviour of the lower troposphere in January and February 1982.
\end{abstract}

Key words. Geomagnetism and paleomagnetism (time variations, diurnal to regular). Meteorology and atmospheric dynamics (general circulation; middle atmosphere dynamics).

\section{Introduction}

The work aims at finding whether there exist distributions of temperature and pressure fields which could be regarded as distributions typical of the given QBO phase and level of geomagnetic activity. In the investigation we followed the paper of Bochníček et al. (1996), which showed that such typical distributions could exist for the particular examples selected. But this work differs from the quoted paper not only in seeking the distributions by means of composite maps and in analysing the pressure fields as well as the temperature fields but also in using the data set on which the paper is based. The result is an original, so far not published, set of temperature and pressure composite maps. In connection with the level of geomagnetic activity and QBO phase the set of temperature and pressure composite maps shows evident differences.

The problems investigated belong to the category of "extraterrestrial influences - weather" research. Most

Correspondence to: J. Bochníček studies describing or explaining those relationships has been limited so far to the analysis of the influence only of one or two selected characteristics of solar activity (Taylor, 1986). The fact that the influence of geomagnetic activity has been underestimated in the past, was probably caused by the opinion that the solar and the geomagnetic activity were so closely correlated that the study of the influence of geomagnetic activity necessarily appeared superfluous. That this is not the case, especially if we correlate two-month averages of the two activities, was shown by Bochníček et al. (1996).

However this work is not the only attempt in this direction, for others including Roberts and Olson (1973) and Wilcox et al. (1973), Bucha (1976, 1993), Pýcha et al. (1992), Bochníček and Pýcha (1994), Bochníček et al. (1996), indirectly Tinsley and Heelis (1993) and Tinsley (1996 a,b), in particular showed that geomagnetic activity can be a factor which, under certain conditions, might influence the behaviour of the lower atmosphere.

\section{Data sources and processing}

Surface air pressure and 700-mb temperature in winter periods 1952-1996 were analysed. The winter period is represented by January and February only. The monthly mean pressure and temperature data were taken from the NCAR Research Set ds195.5 version III, extended. Pressure and temperature deviations for each month were computed as a difference between long term (January, February 1952-1996) and monthly averages.

The winters were classified as being in the east or west phase of the QBO according to the wind direction in January at 50 and $40 \mathrm{mb}:(50+40 \mathrm{mb}) / 2$ (Van Loon and Labitzke, 1988). The data for the equatorial stratospheric wind velocity were taken from Tinsley and Heelis (1993) and Naujokat (Private communication 1996).

Indices of geomagnetic activity $\mathrm{Kp}$ (sum $\mathrm{Kp}$-monthly mean of daily sums of $\mathrm{Kp}$ ) were taken from WDC-A Boulder, Colorado. 
The years analysed (1952-1996) are listed in Table 1, together with indices of geomagnetic activity and QBO phase. The data (1952-1990) were already published by Bochníček et al. (1996) and are presented here in order to have a full picture.

\section{The geomagnetic activity and the distribution of temperature and pressure fields}

In view of the problem connected with the statistical estimate of correlation maps, see Bochníček et al. (1996), and of the fact that the character of meteorological fields at the extreme values of geomagnetic activity, had shown clear elements of congruence, it was decided to use composite maps for the analysis of the influence of geomagnetic activity on the tropospheric changes.

The composite maps of the pressure situations as well as the composite maps of temperature and pressure deviations from the average of the winter periods 1952 1996 were designed so that the corresponding meteorological data were first divided into two groups according to the QBO phase, so that one group included the winter periods where the QBO had the west phase, the second included those where the QBO had the east phase. Then from each of the two groups a set of five periods where geomagnetic activity was the highest was selected and another set of five periods where geomagnetic activity was the lowest. In total, four sets of five winter periods for pressure and temperature situations were selected. These sets of five winter periods are given in Table 2. In Table 2 the year $1982(\mathrm{QBO}$-east; sum Kp $=24.8)$ was not included, because in the course of that winter period geomagnetic activity was considerably unbalanced. Low to medium occurred at the beginning of winter in January ( $\operatorname{sum} \mathrm{Kp}=17.9$ ), when the character of winter is to a large extent being shaped (Kodera, 1991; Kodera and Yamazaki, 1994), and very high activity in February ( $\operatorname{sum} \mathrm{Kp}=31.6)$, that is at the time when the character of the winter period is as a rule already shaped.

Dominant barometric systems in the winter periods over the Northern Hemisphere are represented by the extensive Siberian anticyclone and the lows with their centres over Iceland and Aleutian region. If we examine the character of these formations in connection with QBO phase and the level of geomagnetic activity we will find the Siberian anticyclone remains almost independent of these parameters. However, the Icelandic and
Table 1. The mean values of geomagnetic activity sum $\mathrm{Kp}$ for January, February and winter (January-February), and phase of the quasi-biennial oscillation (QBO)

\begin{tabular}{lllll}
\hline Year & sumKp & & & \\
\cline { 2 - 4 } & January & February & Winter & \\
\hline 1952 & 23.9 & 27.1 & 25.5 & QBO-phase \\
1953 & 20.4 & 19.0 & 19.7 & East \\
1954 & 14.7 & 21.8 & 18.3 & West \\
1955 & 15.9 & 18.1 & 17.0 & West \\
1956 & 22.2 & 18.5 & 20.3 & East \\
1957 & 19.6 & 20.8 & 20.2 & West \\
1958 & 20.8 & 26.6 & 23.7 & West \\
1959 & 19.1 & 25.8 & 22.5 & East \\
1960 & 19.7 & 19.7 & 19.7 & West \\
1961 & 17.2 & 19.1 & 18.1 & East \\
1962 & 10.9 & 15.5 & 13.2 & West \\
1963 & 14.2 & 12.4 & 13.3 & East \\
1964 & 16.5 & 17.7 & 17.1 & West \\
1965 & 11.0 & 14.9 & 12.9 & West \\
1966 & 11.8 & 13.3 & 12.6 & East \\
1967 & 14.2 & 14.6 & 14.4 & West \\
1968 & 18.2 & 20.8 & 19.5 & West \\
1969 & 13.6 & 18.2 & 15.9 & East \\
1970 & 12.8 & 11.6 & 12.2 & West \\
1971 & 17.3 & 17.5 & 17.4 & East \\
1972 & 18.2 & 15.9 & 17.0 & West \\
1973 & 21.2 & 23.7 & 22.4 & East \\
1974 & 20.5 & 20.9 & 20.7 & West \\
1975 & 20.4 & 24.1 & 22.3 & East \\
1976 & 18.9 & 23.0 & 20.9 & West \\
1977 & 16.3 & 17.8 & 17.0 & East \\
1978 & 17.8 & 21.2 & 19.5 & West \\
1979 & 22.1 & 20.3 & 21.2 & West \\
1980 & 16.0 & 15.9 & 15.9 & East \\
1981 & 15.9 & 19.0 & 17.4 & West \\
1982 & 17.9 & 31.6 & 24.8 & East \\
1983 & 21.0 & 26.0 & 23.5 & West \\
1984 & 19.3 & 21.6 & 20.5 & East \\
1985 & 20.9 & 20.2 & 20.6 & East \\
1986 & 18.5 & 24.4 & 21.5 & West \\
1987 & 14.4 & 16.3 & 15.4 & East \\
1988 & 17.2 & 18.6 & 17.9 & West \\
1989 & 23.7 & 21.7 & 22.7 & West \\
1990 & 20.9 & 26.2 & 23.4 & East \\
1991 & 14.7 & 17.2 & 15.9 & West \\
1992 & 20.8 & 26.6 & 24.1 & East \\
1993 & 22.7 & 20.7 & 21.7 & West \\
1994 & 20.7 & 27.9 & 24.3 & West \\
1995 & 17.4 & 18.6 & 18.0 & West \\
1996 & 12.8 & 15.8 & 14.3 & West \\
\hline & & & &
\end{tabular}

Table 2. Winter periods used for the composite maps calculations

\begin{tabular}{|c|c|c|c|c|c|c|c|}
\hline \multicolumn{4}{|c|}{ Low sum Kp } & \multicolumn{4}{|c|}{ High sum Kp } \\
\hline 1962 & 13.2 & 1963 & 13.3 & 1958 & 23.7 & 1952 & 25.5 \\
\hline 1965 & 12.9 & 1966 & 12.6 & 1983 & 23.5 & 1973 & 22.4 \\
\hline 1967 & 14.4 & 1969 & 15.9 & 1989 & 22.7 & 1975 & 22.3 \\
\hline
\end{tabular}


Aleutian low alternatively deepen and fill with $\mathrm{Kp}$ high and low respectively, particularly during the QBO-east phase.

High geomagnetic activity is associated with the deepening of the Icelandic low independent of QBO phase and the filling of the Aleutian low at QBO-east phase.

Low geomagnetic activity is associated with the filling of the Icelandic low independent of QBO phase and the deepening of the Aleutian low at QBO-east phase (see Fig. 1).

If, in connection with geomagnetic activity and QBO phase, we analyse the distribution of pressure deviations from a long-term average (1952-1996), it can be seen, as is evident from Fig. 2, that the distribution of deviations which corresponds to the individual QBO phase differs at different levels of geomagnetic activity. Considerable differences can be found at QBO-east between the distributions at high and low geomagnetic activities and at low geomagnetic activity between QBO-east and QBO-west. At the high sum Kp, the medium and high latitudes are an extensive, continuous area of negative deviations with the largest negative deviation over the North Atlantic and Scandinavia at QBO-east and QBOwest. The secondary extreme is over the Barents Sea at QBO-west, surrounded by two isolated areas of positive deviations. The first with maximum over the Pyrenean Peninsula and northern Africa, the second with the maximum over the northwest Pacific. This arrangement of pressure fields leads to advection of relatively warm air masses over the American and European continents and relatively cold air masses over the northwest Atlantic. At low sum Kp, QBO-east the distribution of positive and negative deviations is turned almost mirrorlike towards the situation of high sum Kp, QBO-east. A difference similar in character although not so conspicuous, exists between high sum Kp, QBO-west, and low sum Kp, QBO-west.

If we analyse the distribution of temperature deviations, we see (Fig. 3) that at the Euro-Asian continent the relatively mild winter periods are associated with high geomagnetic activity, this being so, regardless of the QBO phase. Nevertheless at high sum Kp, QBO-east the warming of the Euro-Asian continent shows so strongly that the temperature deviations in middle to eastern Europe can reach as much as $+2{ }^{\circ} \mathrm{C}$ at $700 \mathrm{mb}$, which corresponds to almost $+3-4{ }^{\circ} \mathrm{C}$ at the surface. With this constellation of conditions (geomagnetic activity $\times$ QBO phase) it is also possible to observe warming in the USA and a relative cooling in the northwest Atlantic and the Aleutian region. Low sum $\mathrm{Kp}, \mathrm{QBO}$-east is associated with a relatively cold winter period in Europe (with a deviation of more than $-1{ }^{\circ} \mathrm{C}$ at $700 \mathrm{mb}$ in northeast Europe, which corresponds to the deviation of $-2-3{ }^{\circ} \mathrm{C}$ at the surface) and in the northern part of Asia and with a relatively warm winter period over Greenland and eastern Canada. Low sum $\mathrm{Kp}, \mathrm{QBO}-$ west is connected with a mildly subnormal temperature in the middle to eastern part of Canada and eastern Europe and with mildly supranormal temperature in northeastern Siberia.
Interesting connections among the level of geomagnetic activity, the QBO phase and the distribution of meteorological fields can be deduced from the pressure and temperature changes, which occurred in the course of the winter period of 1982. In that period, low to medium levels of geomagnetic activity in January was succeeded by a very high level of geomagnetic activity in February. The pressure and temperature situations of that winter period (1982, sum $\mathrm{Kp}=24.8$, QBO-east) were compared with composite maps of high sum $\mathrm{Kp}$, QBO-east. Figure 4 clearly shows the difference in the arrangement of dominant barometric system in the months of January and February 1982. While in January the arrangement of dominant pressure system was different from the situation, which in the case of high sum Kp, QBO-east should be typical (the deepening of the Icelandic low pressure), in February, when average geomagnetic activity was very high (sum $\mathrm{Kp}=31.6$ ), we could observe considerable deepening of the Icelandic low.

The behaviour of pressure deviations (see Fig. 5) indicates similar features. Clearly there were differences in January, while in February the distribution of positive and negative pressure deviations regrouped into the formation which was by its character similar to that of high sum Kp, QBO-east.

In the distribution of temperature deviations (see Fig. 6) no such clear analogy between the temperature situation of February 1982 and high sum Kp, QBO-east was found. Nevertheless some elements of congruence can be found there too.

\section{Possible mechanisms}

The most important problem in "extraterrestrial influences - weather" research is to find mechanisms that could reliably explain the phenomena described. A great number of attempts in this direction have been made (Charney and Drazin, 1961; Dickinson, 1968; Geller, 1981, 1983; Mayr and Harris, 1981; Plumb, 1989; Kodera, 1991; Kodera et al. 1991; Tinsley and Deen, 1991; Tinsley and Heelis, 1993; Tinsley, 1996a,b). The individual mechanisms can be assigned to three main groups, the group of dynamic mechanisms (planetary waves), radiation mechanisms (temperature unbalance in the middle atmosphere) and electric mechanisms (modulation of global electrical circuit). Even though the electrical mechanism is at the present time the best worked out and appears to be the most effective in terms of energetics, the influence of the other mechanisms cannot be neglected. One of the possible combinations of the simultaneous action of more mechanisms is shown in Fig. 7. More precision about these relations can be obtained only if "extraterrestrial influences $\times$ weather" research is carried out in substantially shorter time intervals, i.e. a number of days. Such an approach will make it possible to examine the effectiveness of tropospheric response of the individual, exactly defined magnetospheric phenomena. 
High Sum $\mathrm{Kp}$ - west

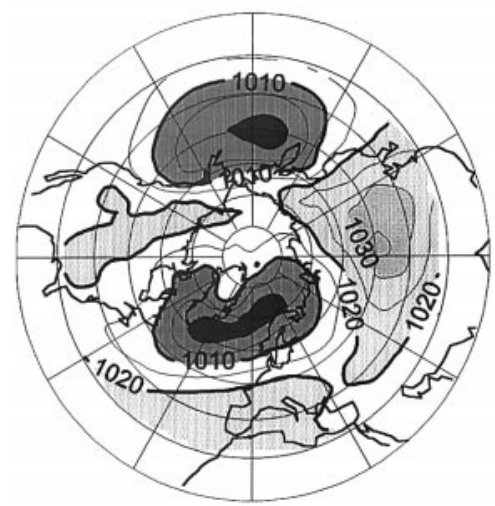

Low Sum Kp - west

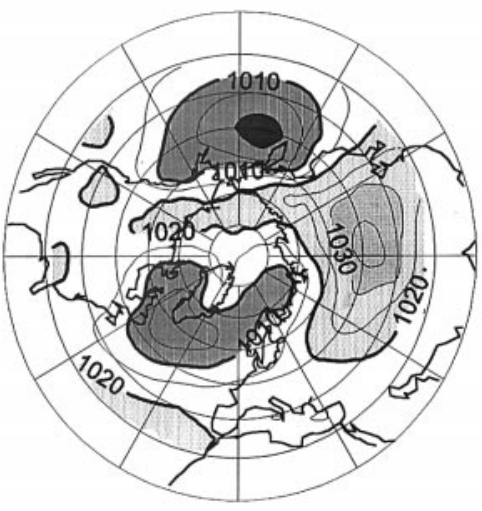

High Sum Kp - west

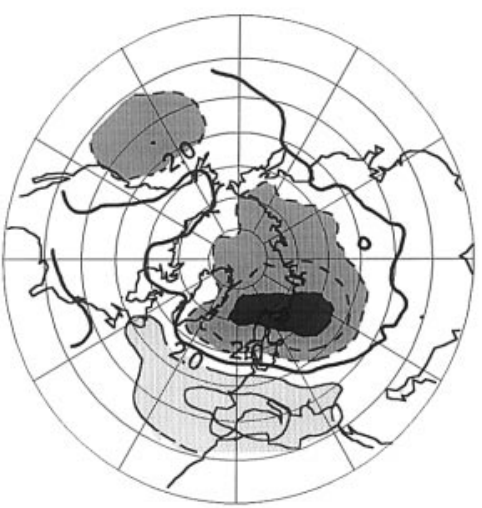

Low Sum Kp - west

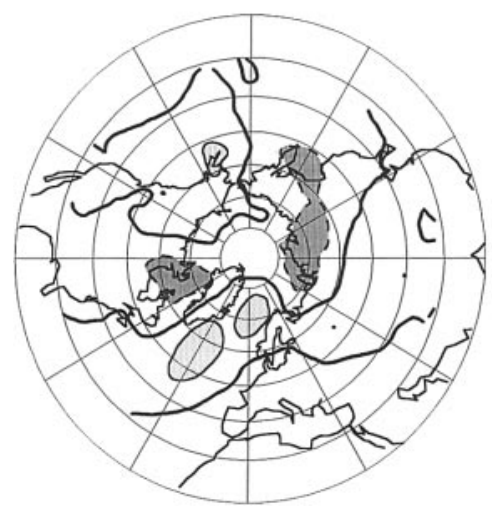

High Sum Kp - east

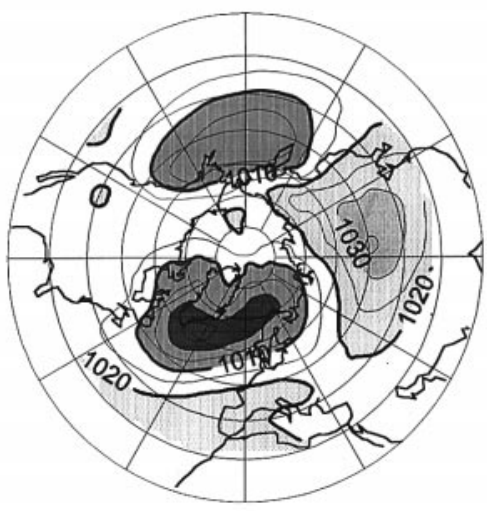

Low Sum Kp - east

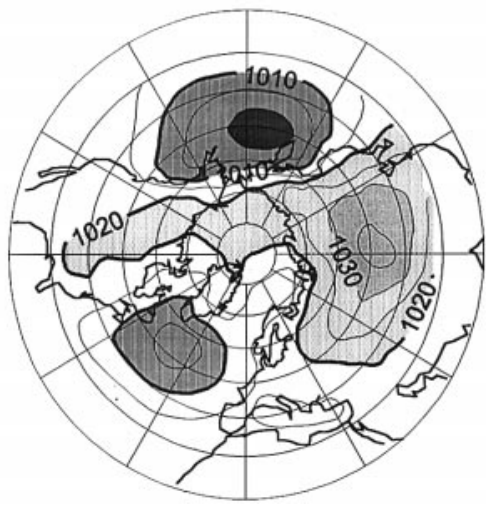

High Sum Kp - east

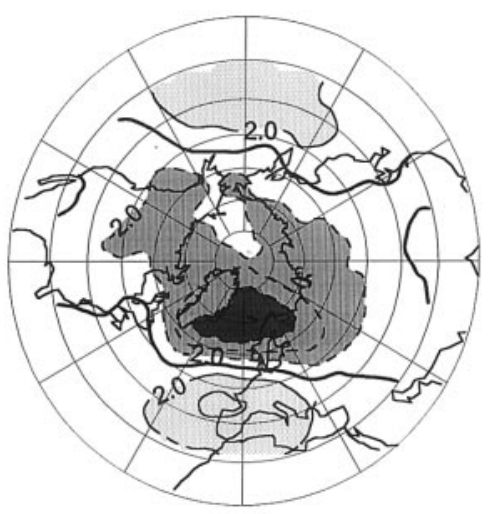

Low Sum Kp - east

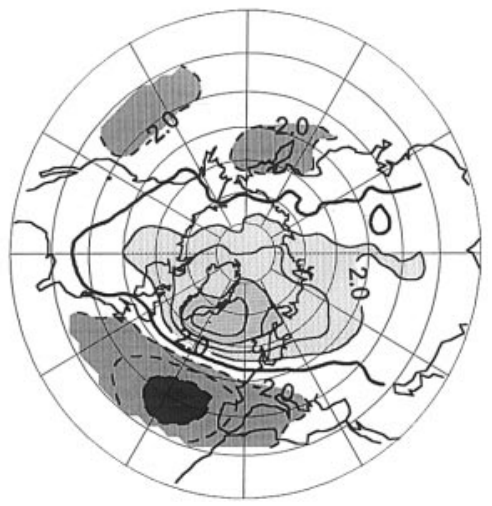

Fig. 1. Winter period dominant barometric systems over the Northern Hemisphere. The Siberian anticyclone remains almost independent of the level of geomagnetic activity and QBO-phase. The deepening of the Icelandic and Aleutian low alternate with high and low Kp values and QBOeast phase. The intervals between contours are $5 \mathrm{mb}$
Fig. 2. Distribution of pressure deviations from a long term average (1952-1996) at different levels of geomagnetic activity and at different QBOphases (positive, solid lines; negative, dashed lines). The intervals between contours are $2 \mathrm{mb}$ 

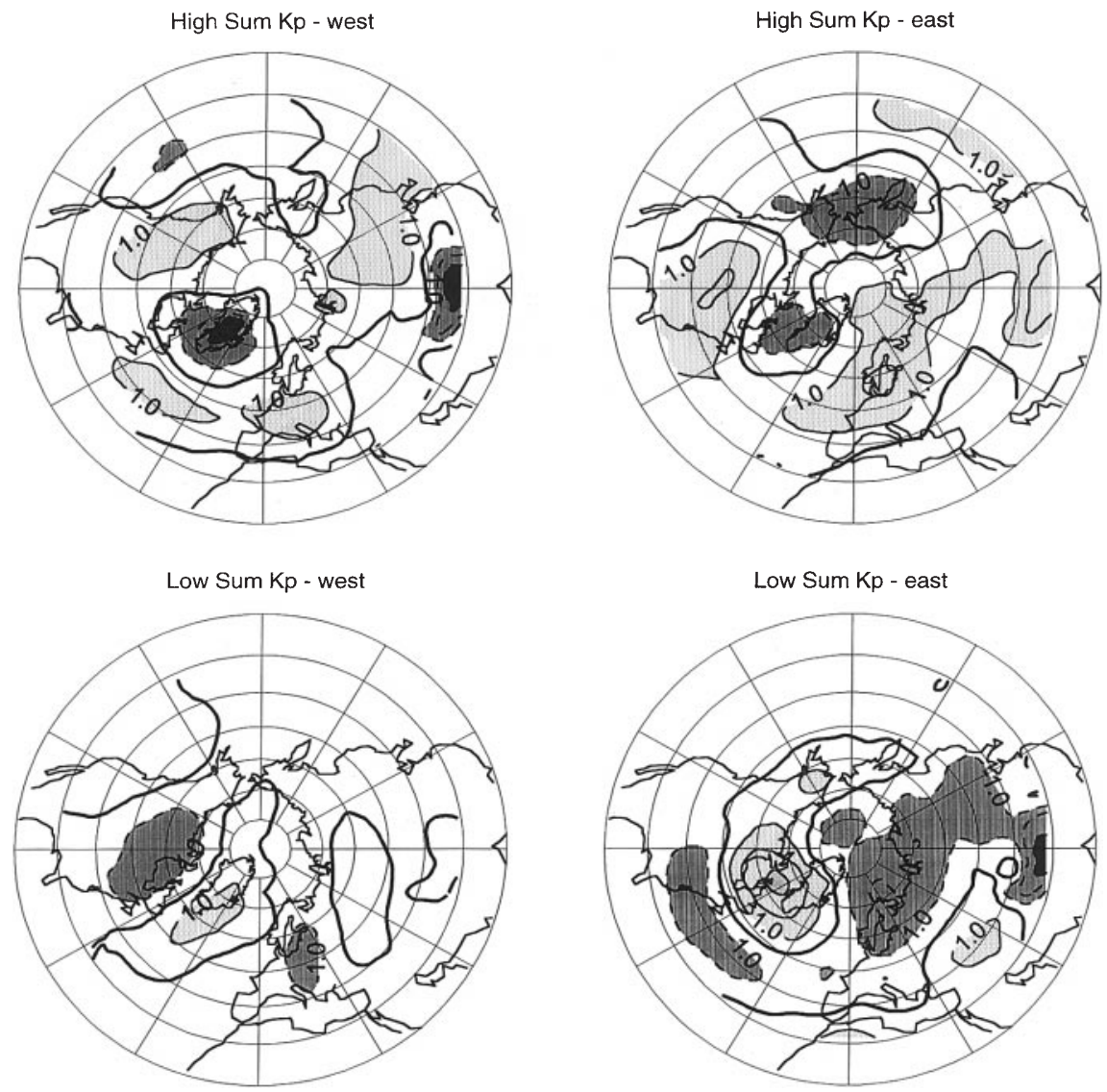

Fig. 3. Distribution of temperature deviations from a long term average (1952-1996) at different levels of geomagnetic activity and at different QBO-phases (positive, solid lines; negative, dashed lines). The intervals between contours is $1^{\circ} \mathrm{C}$

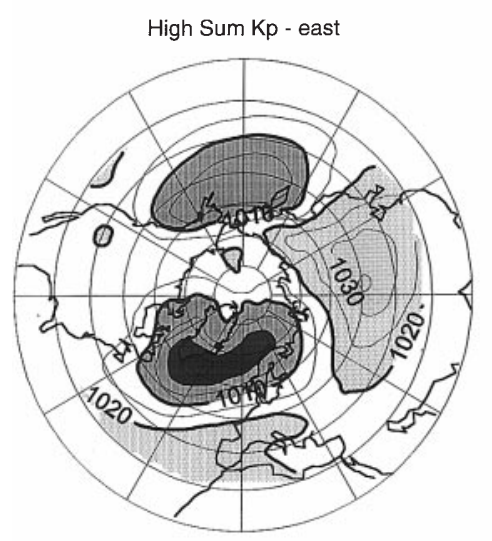

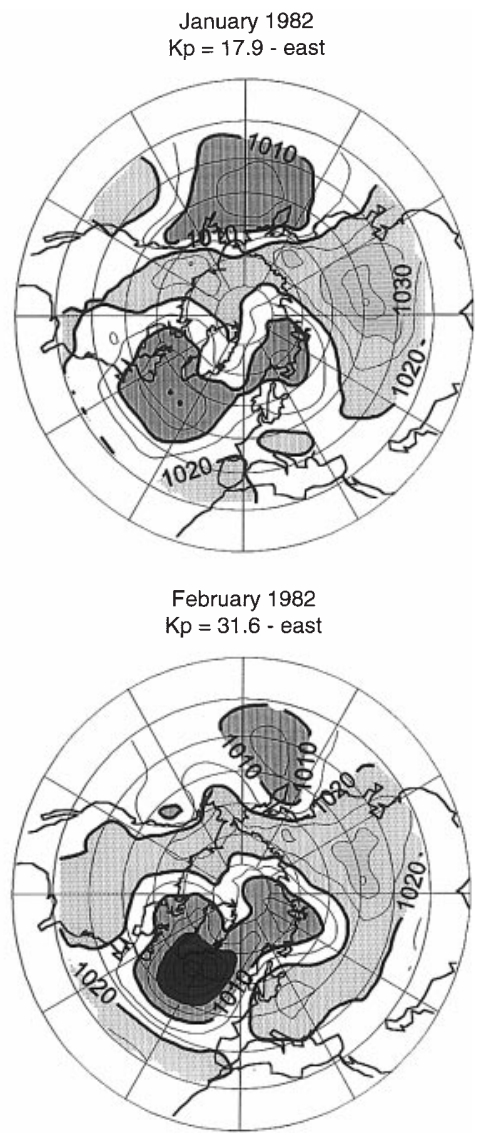

Fig. 4. Dominant pressure system in individual months of winter period 1982 in comparison with the composite map of dominant pressure system at high sum Kp, QBO-east. The intervals between contours are $5 \mathrm{mb}$ 


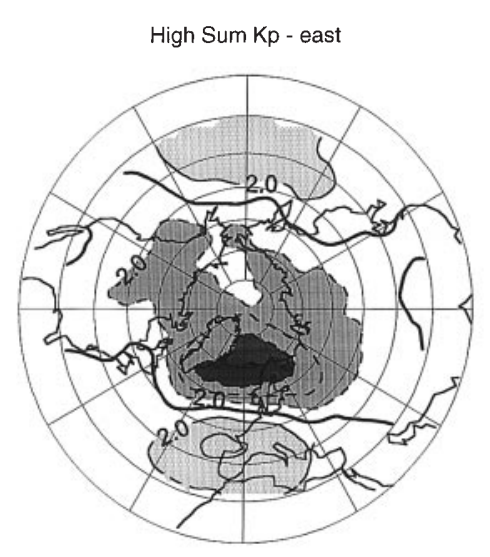

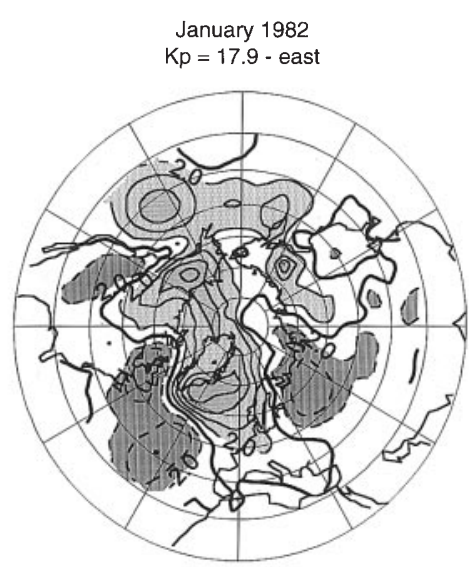

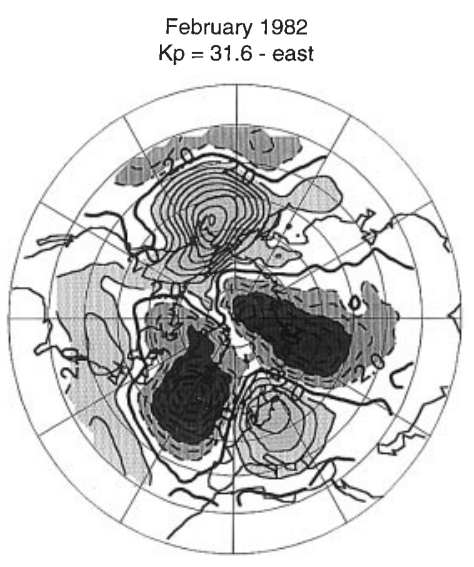

Fig. 5. Distribution of pressure deviations in individual months of winter period 1982 in comparison with composite map of pressure deviations at high sum Kp, QBO-east. The intervals between contours are $2 \mathrm{mb}$
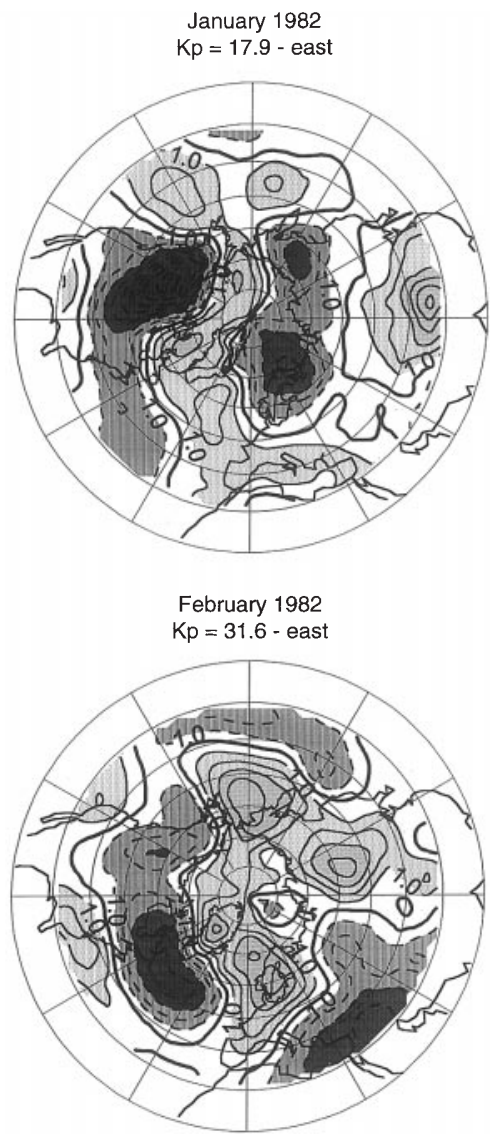

Fig. 6. Distribution of temperature deviations in individual months of winter period 1982 in comparison with the composite map of temperature deviations at high sum $\mathrm{Kp}$, QBO-east. The intervals between contours is $1^{\circ} \mathrm{C}$ 


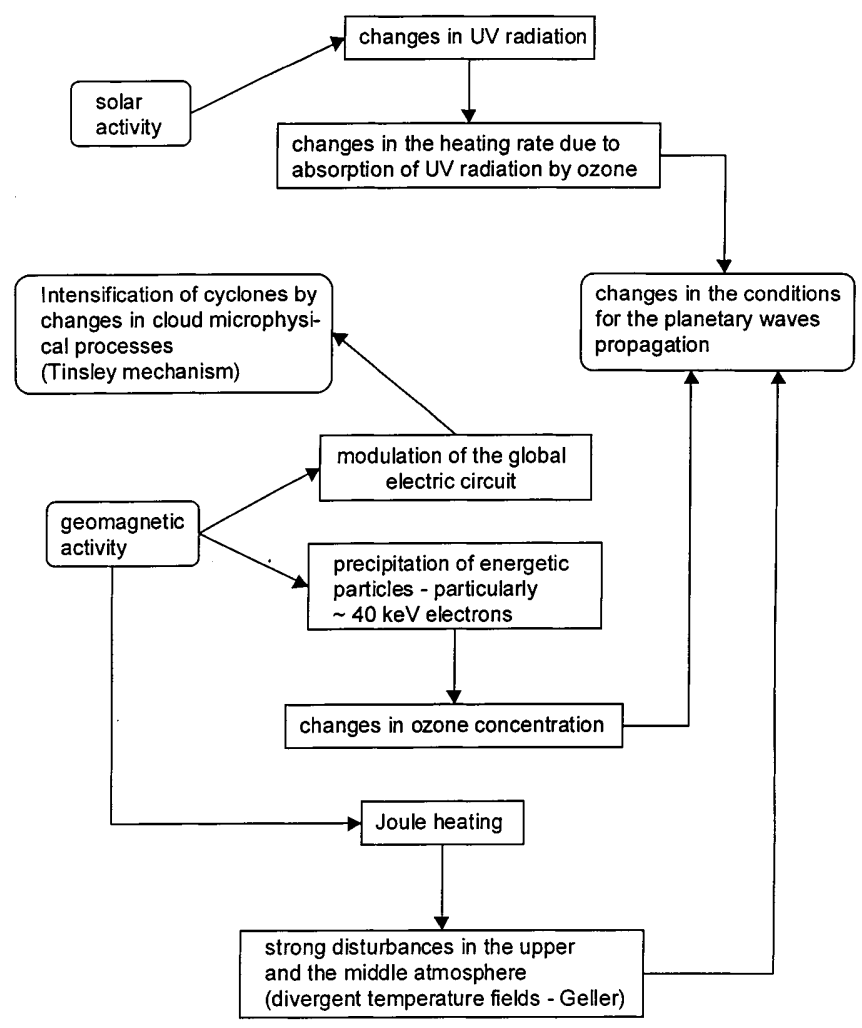

Fig. 7. A possible combination of simultaneous action of more mechanisms on processes in the troposphere

\section{Conclusions}

The results of the work presented can be summed up into the following scheme:

\section{High sum Kp QBO-west}

a. Aleutian low pressure is deep

b. Icelandic low pressure deepens

c. Extensive, continuous areas of negative pressure deviations with the main maximum over the North Atlantic and Scandinavia and the secondary maximum over the Barents Sea, surrounded by isolated areas of positive deviations

d. Warm air over USA and Euro-Asian continent, cold air over Greenland

\section{Low sum Kp $Q B O$-west}

a. Aleutian low pressure is deep

b. Icelandic low pressure fills

c. Distribution of pressure deviations split into isolated areas of positive and negative deviations d. Cold air over Canada and eastern Europe, warm air over Greenland

d. Cold air over eastern part of USA and Euro-Asian continent, warm air over Canada and Greenland

Acknowledgements. This work was supported by grant A3012806/ 1998 of the Grant Agency of the Academy of Sciences of the Czech Republic.

Topical Editor F. Vial thanks M. A. Shea and K. Lassen for their help in evaluating this paper

\section{References}

Bochníček, J., and J. Pýcha, Geomagnetic activity as a possible cause of changes in the middle atmosphere and troposphere, Stud. Geophys. Geod., 33, 103-107, 1994.

Bochníček, J., V. Bucha, P. Hejda, and J. Pýcha, Relation between Northern Hemisphere winter temperatures and geomagnetic or solar activity at different QBO phases, J. Atmos. Terr. Phys., 58, 883-897, 1996.

Bucha, V., Variations of the geomagnetic field, the climate and weather, Stud. Geophys. Geod., 20, 149-167, 1976.

Bucha, V., Impact of solar perturbations on changes in the atmospheric circulation, in, The Earth and the Universe, Ed. W. Schröder, Newsletters of the ICH of IAGA, pp. 125-132, 1993.

Charney, J. G., and P. G. Drazin, Propagation of planetary-scale disturbances from the lower into the upper atmosphere, J. Geophys. Res., 66, 83-109, 1961.

Dickinson, R.E., Planetary Rossby waves propagating through weak westerly wind wave guides, J. Atmos. Sci., 25, 984-1002, 1968.

Geller, M. A., Middle atmosphere dynamics and composition, in, Exploration of the Polar Upper Atmosphere, Eds. C. S. Deehr, and J. A. Holtet, D. Reidel, Dordrecht, Holland, pp. 1-16, 1981.

Geller, M. A., Dynamic of the middle atmosphere, Space Sci. Rev., 34, 359-375, 1983.

Kodera, K., The solar and equatorial QBO influences on the stratospheric circulation during the early Northern-Hemisphere winter, Geophys. Res. Lett., 18, 1023-1026, 1991.

Kodera, K., and K. Yamazaki, A possible influence of recent polar stratospheric coolings on the troposphere in the Northern Hemisphere winter, Geophys. Res. Lett., 21, 809-812, 1994.

Kodera, K., M. Chiba, and K. Shibata, A general circulation model study of the solar and QBO modulation of the stratospheric circulation during the Northern Hemisphere winter, Geophys. Res. Lett., 18, 1209-1212, 1991.

Mayr, H. G., and I. Harris, Wind induced composition effects at high latitudes, in, Exploration of the Polar Upper Atmosphere. Eds. C. S. Deehr, and J. A. Holtet, D. Reidel, Dordrecht, Holland, 31-54, 1981.

Naujokat, B., QBO phases in winter intervals 1952-1996 (private communication), 1996.

Plumb, R. A., On the seasonal cycle of stratospheric planetary waves, PAGEOPH, 130, 233-242, 1989.

Pýcha, J., P. Hejda, and J. Bochníček, A possible relation between geomagnetic activity and winter air surface temperature in Prague, Stud. Geophys. Geod., 36, 329-337, 1992.

Roberts, W. O., and R. H. Olson, Geomagnetic storms and wintertime $300 \mathrm{mb}$ through development in the North Pacific-North America area, J. Atmos. Sci., 30, 135-140, 1973.

Taylor, Jr. H. A., Selective factors in Sun-weather research, Rev. Geophys., 24, 329-348, 1986.

Tinsley, B. A., Solar wind modulation of the global electric circuit and apparent effects on cloud microphysics, latent heat release, and tropospheric dynamics, J. Geomag. Geoelectr., 48, 165-175, 1996a.

Tinsley, B. A., Correlations of atmospheric dynamics with solar wind-induced changes of air-earth current density into cloud tops, J. Geophys. Res., 101, 29 701-29 714, 1996b. 
Tinsley, B. A., and G. W. Deen, Apparent tropospheric response to $\mathrm{MeV}-\mathrm{GeV}$ particle flux variations: a connection via electrofreezing of supercooled water in high-level clouds?, J. Geophys. Res., 96, 22 283-22 296, 1991.

Tinsley, B. A., and R. A. Heelis, Correlations of atmospheric dynamics with solar activity: evidence for a connection via solar wind, atmospheric electricity, and cloud microphysics, J. Geophys. Res., 98, 10 375-10 384, 1993.
Van Loon, H., and K. Labitzke, Association between the 11-year solar cycle, the QBO, and the atmosphere. Part II: surface and $700 \mathrm{mb}$ in the Northern Hemisphere in winter, J. Clim., 1, 905920, 1988.

Wilcox, J. M., P. H. Scherrer, L. Svalgaard, W. O. Roberts., and R. H. Olson, Solar magnetic structure relation to circulation of the Earth's atmosphere, Science, 180, 185-186, 1973. 